\title{
Hydrovoltaic cells. Part II: Thermogalvanic cells and numerical simulations of thermal diffusion potentials
}

\author{
Jacques Josserand, Valérie Devaud, Grégoire Lagger, Henrik Jensen, Hubert H. Girault * \\ Laboratoire d'Electrochimie Physique et Analytique, Institut de Chimie Moléculaire et Biomoléculaire, \\ Ecole Polytechnique Fédérale de Lausanne, Lausanne CH-1015, Switzerland
}

Received 10 April 2003; received in revised form 29 August 2003; accepted 25 September 2003

\begin{abstract}
When two electrolyte solutions of different temperature are placed in contact, a thermal diffusion potential (TDP) is established. The phenomenon is studied numerically using finite element simulations of the temperature distribution within a hydrodynamic cell. Experimentally, the hydrovoltaic flow cell is used to demonstrate how a temperature difference can induce redox reactions at electrodes placed in the two liquids in order to extract a current continuously in an external circuit resulting in a power-generating unit. When the concentration of the redox couple introduced in the solution is moderated, it is shown that the TDP is not negligible, even if the main driving force is due to the temperature effect on the standard potential of the couple present. The numerical model may also be applied in more general situations involving thermal effects in microsystems.
\end{abstract}

(C) 2003 Elsevier B.V. All rights reserved.

Keywords: Thermogalvanic system; Thermal diffusion potential; Energy conversion; Numerical model; Finite element simulations; Heat transfer

\section{Introduction}

A temperature gradient between two electrolyte solutions of similar concentrations leads to a gradient in the chemical potential, which depends on the magnitude of the heats of transport of the ions constituting the electrolyte. The heat of transport of a given ion is simply the energy required to transport the ion between regions of different temperature [1]. The heat of transport of individual ions cannot be measured directly, therefore a reference ion is chosen and from the measurable heats of transport of salts, values of relative ionic heats of transport can be obtained. Usually the heat of transport of salts is positive, meaning that most salts transfer from hot to cold regions in response to a temperature gradient. If we consider an electrolyte solution of uniform concentration, the result of an imposed temperature gradient is therefore normally a flux of electrolyte from \footnotetext{
67.

${ }^{*}$ Corresponding author. Tel.: +41-21-693-31-51; fax: +41-21-693-36-

E-mail address: hubert.girault@epfl.ch (H.H. Girault).
}

hot to cold (this phenomenon is termed the Soret effect). The thermal mobilities (i.e., the rates of mass transfer for a given temperature gradient in the absence of an external electric field) of the anion and cation in the electrolyte are different due to differences in their ionic mobilities and heats of transport, but they have to transfer at the same rate to ensure internal electroneutrality of the solution. Consequently, a potential difference, termed a thermal diffusion potential (TDP), is established quasi-instantaneously in response to a temperature gradient in order to speed up the ion with the lower thermal mobility and slow down the ion having the higher thermal mobility. This phenomenon has for instance been quantified in the case of immiscible liquids in order to measure ionic transport entropies [2,3].

It is important to note that the TDP should not be confused with a diffusion potential, which is solely established as a result of an electrolyte concentration gradient rather than a temperature gradient. However, in situations where an electrolyte concentration gradient is generated over time in response to a temperature gradient, a diffusion potential opposing the TDP will eventually be established. 
The generation of electricity between two laminar flowing solutions (i.e., without a membrane to separate the solutions) has previously been demonstrated by using different redox couples in the liquids [4-6] or by taking advantage of a diffusion potential established due to an electrolyte concentration gradient [7]. In this work, we shall illustrate by numerical simulations how a temperature gradient can induce a TDP when the species have different thermal mobilities. For moderate values of the redox couple concentration, this potential difference can also represent a non-negligible component of the potential induced in a thermogalvanic cell (where the redox reaction is driven by the variation of the standard potential with temperature). Such a thermogalvanic cell can be used to extract energy from two solutions of different temperatures flowing in contact, as in previous work dedicated to convective cells $[6,8,9]$. In contrast, other studies have rather focused on static thermogalvanic cells [9-11]. This subject is important as practical methods for extracting the low-grade excess energy from solutions having modulated temperatures are in high demand [12].

Concerning the numerical aspects, simulations of heat transfer applied to flowing microsystems have been frequently addressed in past years [13-24] but the TDP has not been treated under such conditions. In this paper, the temperature distributions and TDP are analysed using finite element simulations of a flow cell under open circuit conditions and are compared with experimental results. This model follows a previous simulation of the concentration diffusion potential in microsystems [25]. The experimental investigation is extended to include the effects of external electrical loads, redox couple concentration, flow rate and electrode surface.

\section{Theory}

When a temperature gradient is applied across an electrolyte solution of uniform concentration, an enrichment of electrolyte in one region (usually the cold one) ensues. At a certain point a steady state is reached and a concentration gradient given by [1]

$\frac{1}{m}=\operatorname{grad} m=\sigma \operatorname{grad} T$

has been established. In this equation $m$ is the molality of the electrolyte solution, $T$ is the temperature and $\sigma$ is the Soret coefficient. The Soret coefficient, as defined by Eq. (1), can be regarded as a proportionality factor that links mass transport and thermal diffusion.

Immediately after the temperature gradient is imposed, and thus prior to steady state, a pure thermal diffusion potential is established according to the following equation [2]:
$F \operatorname{grad} \phi=-\sum_{k} \tau_{k}\left(\left(\operatorname{grad} \mu_{k}\right)_{T, p}+\frac{{ }^{*} Q_{k}}{T} \operatorname{grad} T\right)$,

where $\tau_{k}=t_{k} / z_{k}$ is the reduced transport number $\left(t_{k}\right.$ is the Hittorf transport number), ${ }^{*} Q_{k}$ is the heat of transport and $\mu_{k}$ is the chemical potential. The summation is carried out on all the ions, $k$, in solution. The term $\left(\operatorname{grad} \mu_{k}\right)_{T, p}$ is simply the gradient of the chemical potential at constant temperature and pressure. The heat of transport is related to the transport entropy according to the following definition:

${ }^{*} Q_{j}=T\left({ }^{*} S_{j}-S_{j}\right)$,

where ${ }^{*} S_{j}$ is the transport entropy and $S_{j}$ is the partial molar entropy.

Eq. (2) describes the thermal diffusion potential, which is established when a hot solution comes into contact with a cold solution in a flow cell as described in Fig. 1. Except for a few sporadic reports, relatively few studies have been reported on this topic [1-3]. The thermal diffusion potential between point 2 and 3 in Fig. 1 can be approximated by

$F\left(\phi_{3}-\phi_{2}\right)=-\sum_{k} \tau_{k} \Delta_{2}^{3}\left(\mu_{k}^{0}\right)_{T, p}-\sum_{k} \frac{\tau_{k}^{*} Q_{k}}{T} \Delta T$,

where $\Delta T=T_{3}-T_{2}$. In situations where a concentration gradient is not present (as in this work), the first term in Eq. (4) can be omitted, leading to

$F\left(\phi_{3}-\phi_{2}\right)=-\sum_{k} \frac{\tau_{k}^{*} Q_{k}}{T} \Delta T$

Obviously, if the temperature gradient acts on the system for a long time, a concentration gradient is gener-

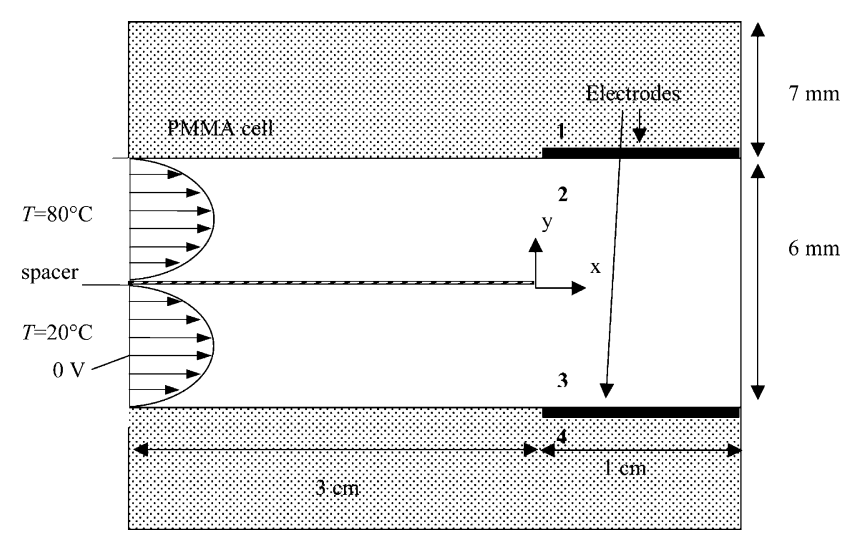

Fig. 1. Schematic illustration of the flow cell (not to scale), geometry and boundary conditions used for the three-step numerical simulation (fluidic, thermal and potential-concentration). Points 1 and 4 are placed on the electrodes to describe the contribution given by the temperature variation of the redox couple standard potential (Eq. (6)). The initial concentrations $c_{1}$ and $c_{2}$ have a uniform distribution of $1 \mathrm{mM}$. The simulated TDP results are independent of the $\mathrm{NaCl}$ concentration because the migration and thermal diffusion terms of Eq. (11) (which equilibrate themselves for electroneutrality) are both proportional to this concentration. 
ated according to Eq. (1). When a concentration gradient exists, the overall potential difference is composed of the TDP and a concentration diffusion potential. However, at this point we shall be concerned only with the situation where the two liquids are in contact for a very short time and consequently no concentration gradient is present in the system.

In the present cell the ferri/ferrocyanide redox couple is present in both flowing solutions. Since the redox potential of the redox couple is temperature dependent, it will not have similar standard potential values at the hot electrode 1 and the cold electrode 4 . In other words, the measured potential at open circuit between 1 and 4 is the sum of a thermal diffusion potential given by Eq. (5) and the temperature effect on the redox potential of the redox couple. In the present set-up, the overall measured potential difference, $\Delta E$, can thus be expressed as:

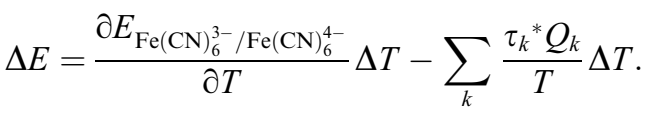

The contribution from the thermal diffusion potential can be calculated to be $11.3 \mathrm{mV}$ for a temperature difference of $60{ }^{\circ} \mathrm{C}$ if it is assumed that only sodium chloride contributes $[1,12]$.

\section{Numerical model for thermal diffusion potential}

\subsection{Equations}

The open circuit configuration is treated using three independent calculations: (i) the fluid velocity distribution (to be injected in the two following steps), (ii) the temperature convection-conduction (including the external heat exchanges of the cell by natural convection and radiation) and (iii) the diffusion-migration of the species (including convection) which relies on the results of (i) and (ii). The hot and cold fluids (typically $80-20{ }^{\circ} \mathrm{C}$ for the reference case) are injected at the respective "up and down" inlets of the channel in laminar conditions. A typical value of the outlet Reynolds number is 30 ( $R e=\bar{V} w / v$, where $v$ is the kinematic viscosity of the fluid $\left(10^{-6} \mathrm{~m}^{2} / \mathrm{s}\right), w$ is the width of the channel after the junction $(6 \mathrm{~mm})$ and $\bar{V}$ is the mean value of the fluid velocity in the channel, $\bar{V}=5.2 \mathrm{~mm} \mathrm{~s}^{-1}$ ). The steadystate Navier-Stokes equation (6) and the continuity equation (7) are solved in the case of an incompressible fluid flowing in a horizontal direction (no gravity effect) in laminar conditions [26]:

$$
\begin{aligned}
& \rho \boldsymbol{V} \cdot \nabla \boldsymbol{V}=-\nabla p+\mu \nabla^{2} \boldsymbol{V}, \\
& \nabla \cdot \boldsymbol{V}=0,
\end{aligned}
$$

where $p$ is the pressure, $\rho$ is the density, $\mu$ is the dynamic viscosity of the fluid and $\boldsymbol{V}$ is the velocity vector of the flowing solution. The variation of $\mu$ and $\rho$ with tem- perature and species concentration is neglected in this step to decouple the fluid velocity field from the other unknowns and thus to calculate it in an independent step. It should be noted that $\mu$ is sensitive to temperature variations (its value divided by 3 approximately from 20 to $80^{\circ} \mathrm{C}$ ), but this assumption can be made because the Poiseuille velocity profile is independent of the viscosity (in each flow inlet). However, it is clear that this remains a first-order approximation for the region of temperature gradient.

The model validity also requires that the pumping system deliver the same flow rates in both inlets whatever the temperature difference. The natural convection effect has been minimised experimentally by infusing the hot solution in the upper part of the channel. For this reason, this effect is neglected in the present model. To justify the 2D assumption of the flow, a large depth over width of the channel is assumed for all the calculations (i.e., a rectangular channel of infinite depth corresponding to a flow between 2 parallel plates). In the experimental case the value of this ratio at the inlets is 3 , which is at the limit of the validity of the $2 \mathrm{D}$ assumption. The flow profile is assumed to be parabolic at the channel inlets (established flow).

The temperature field $T$ is obtained by solving the convection-conduction equation (9), where $\lambda$ and $C_{\mathrm{p}}$ represent the thermal conductivity and the specific heat capacity of the fluid, respectively [27]. Eq. (9) is accompanied by the Fourier condition at the outside walls of the cell (Eq. (10)) to take into account the external exchanges by convection and radiation [27]:

$$
\begin{aligned}
& \frac{\partial T}{\partial t}+\nabla \cdot\left(-\frac{\lambda}{\rho C_{\mathrm{p}}} \nabla T+\boldsymbol{V} T\right)=0, \\
& -\lambda \frac{\partial T}{\partial n}=h\left(T-T_{\mathrm{amb}}\right)+\sigma_{0} \varepsilon\left(T^{4}-T_{\mathrm{amb}}^{4}\right),
\end{aligned}
$$

where $h, \sigma_{0}, \varepsilon$ and $T_{\text {amb }}$ represent, respectively, the convection coefficient at the wall|air interface, the Stephan Boltzmann coefficient, the surface emissivity coefficient and the local ambient temperature. The radiation exchange is assumed to be located at the wall|air interface, even if the cell material is transparent. It is worth remembering that the thermal diffusivity $\lambda / \rho C_{\mathrm{p}}$ is 2 orders of magnitude higher than the typical diffusion coefficient of the species (i.e., $10^{-7}$ compared with $10^{-9} \mathrm{~m}^{2} / \mathrm{s}$ ). As a consequence, the miniaturisation is here limited to the millimeter scale in order to prevent a diffusive mixing that would cancel the temperature gradient. The transient expression of the flux conservation of the species $i$ (the salt anion and cation, respectively) can be written as

$$
\begin{gathered}
\frac{\partial c_{i}}{\partial t}+\nabla \cdot\left(-D_{i} \nabla c_{i}+\boldsymbol{V} c_{i}-\frac{z_{i} F}{R T} D_{i} c_{i} \nabla \phi\right. \\
\left.-\frac{{ }^{*} Q_{i}}{R T^{2}} D_{i} c_{i} \nabla T\right)=0, \quad i=1,2,
\end{gathered}
$$


$z_{1} c_{1}+z_{2} c_{2}=0$

where $c_{i}, D_{i}, z_{i}$ and ${ }^{*} Q_{i}$ stand for the respective concentration, diffusion coefficient, electrical charge and heat of transport in the Hittorf reference system [1]. $F$ and $R$ denote the Faraday constant and the gas constant. The diffusion-migration term (corresponding to the Nernst-Planck equation) is here supplemented with the convection term and the thermal transport [1$3]$. The temperature gradient induces a flux of the electrolyte due to the Soret effect [1]. Consequently, an electric field is generated in order to ensure the electroneutrality condition (Eq. (12)) via the migration term as the two ions have different thermal mobilities. This gives rise to the so-called thermal diffusion potential [1] by analogy to the diffusion potential [7,26,28]. In Eq. (12) the Poisson term has been neglected, due to the limited values of the electric field gradient. The model is based on the diluted solution assumption, assuming equality between the concentrations of the species and their activities.

\subsubsection{Numerical parameters}

All the present simulations have been performed using the finite element commercial software Flux-Expert ${ }^{\circledR}$ (Simulog, 35 Chemin du Vieux Chêne, 38240 Meylan Zirst, France. Contact: anne-marie.bernier@simulog.fr) operated on a SGI Octane 2 Unix workstation (1 Gb RAM). The numerical formulation corresponding to the last set of equations is described in Appendix A whereas the hydrodynamic and thermal equations are standard. All equations are solved in dimensional form and applied to 2D Cartesian geometries. Non-linear algorithms based on the Gauss inversion method are used with a convergence criteria fixed to $0.5 \%$ for the iterative scheme. A steady-state algorithm is used for the hydrodynamic and thermal calculations while a transient calculation is performed for concentration and potential. A typical time-step value for a flow velocity of 10 $\mathrm{mm} \mathrm{s}^{-1}$ is $0.01 \mathrm{~s}$.

The studied geometry is presented in Fig. 1 with the main boundary and initial conditions. For the fluidic calculations, the boundary conditions are the parabolic velocity profiles at the channel inlets and the no slip conditions at the channel walls. For the temperature calculations, the Dirichlet conditions are fixed at the channel inlets $\left(T=80\right.$ and $20{ }^{\circ} \mathrm{C}$ for the upper and lower inlets, respectively). On the external walls of the cell, $T_{\text {amb }}$ is fixed at $20{ }^{\circ} \mathrm{C}$. For the third step (potential and concentration calculations), the zero potential condition (reference value) is fixed at one point on the lower inlet of the channel. On all the other parts of the domain, the electric potential is allowed to act on the species in order to ensure the electroneutrality condition. In the fluidic calculation, parabolic flow profiles are imposed at the inlets of the channel. The finite ele-
Table 1

Parameters used for the calculation of thermal diffusion (i.e., fluid thermal conductivity $(\lambda)$ density $(\rho)$, specific heat capacity $\left(C_{\mathrm{p}}\right)$ and the resulting thermal diffusivity $\left.\left(\lambda / \rho C_{\mathrm{p}}\right)\right)$

\begin{tabular}{|c|c|c|c|c|}
\hline & $\begin{array}{l}\lambda \\
\left(\mathrm{W} \mathrm{m}^{-1} \mathrm{~K}^{-1}\right)\end{array}$ & $\begin{array}{l}\rho \\
\left(\mathrm{kg} \mathrm{m}^{-3}\right)\end{array}$ & $\begin{array}{l}C_{\mathrm{p}} \\
\left(\mathrm{kJ} \mathrm{kg}^{-1} \mathrm{~K}^{-1}\right)\end{array}$ & $\begin{array}{l}10^{7} \lambda \rho^{-1} C_{\mathrm{p}}^{-1} \\
\left(\mathrm{~m}^{2} \mathrm{~s}^{-1}\right)\end{array}$ \\
\hline Water & 0.6 & 1000 & 4.187 & 1.43 \\
\hline PMMA & 0.21 & 1200 & 1.4 & 1.25 \\
\hline $\mathrm{Pt}$ & 70 & 21,500 & 0.13 & 250 \\
\hline
\end{tabular}

ment mesh is described in Appendix B. It has been verified that the mesh elements sizes are sufficiently small to have no significant influence on the results.

All calculations have been performed assuming a parabolic shaped pressure driven flow. The $2.3 R T / F$ value is fixed at $60 \mathrm{mV}$ at $20{ }^{\circ} \mathrm{C}$ and the temperature dependence of the diffusion coefficient is taken into account using the Stokes-Einstein relation [29]. The species charge $z_{1}, z_{2}$ are equal to 1 and -1 , respectively. The diffusion coefficient values are explained in Appendix B. The values of ${ }^{*} Q_{1}$ and ${ }^{*} Q_{2}$ are -4229 and $7118 \mathrm{~J} / \mathrm{mol}$ for $\mathrm{Na}^{+}$and $\mathrm{Cl}^{-}$, respectively [1]. The thermal diffusivities $\lambda / \rho C_{\mathrm{p}}$ are described in Table $1[26,30]$. The convection coefficient at the wall|air interface $h$ is fixed at $10 \mathrm{~W} / \mathrm{m}^{2}$ $\mathrm{K}$, the Stephan Boltzmann coefficient equals $5.67 \times 10^{-8}$ $\mathrm{W} / \mathrm{m}^{2} \mathrm{~K}^{4}$ and the surface emissivity coefficient, $\varepsilon$, is fixed at 0.85 for the polymethylmethacrylate (PMMA) interface. The resulting transversal Biot number of the cell (defined by $h w / \lambda$ ) equals 0.38 and $T_{\text {amb }}$ is $20^{\circ} \mathrm{C}$.

\section{Experimental}

The experimental set-up consisted of a flow cell comprising two platinum electrodes (area $=0.5 \mathrm{~cm}^{2}$ ) positioned face to face on the walls of the flow cell. For the first experiments (open circuit conditions, see Fig. 5), the electrodes were placed near the middle of the channel, i.e., beginning $3 \mathrm{~cm}$ after the fluid inlets. This corresponds to Fig. 1, where the last centimetre of the channel is not represented (this cell design is similar to what has been described in part I of this series [7]). For the other experiments (power generation), the electrodes were placed at the beginning of the channel. The channel length was $5 \mathrm{~cm}$, its width was $2 \times 3 \mathrm{~mm}$ before the fluid junction, and its depth was $1 \mathrm{~cm}(d / w=3.33$ before the junction). A film was located between the two halves of the cell to act as a spacer. For the first experiments and the simulations, it was a $60 \mu \mathrm{m}$ polymer film. For the power measurements, the spacer used was a $1 \mathrm{~mm}$ silicon film. This spacer was opened in the middle of the cell, determining the contact surface between the two solutions. The contact surface was equal to the surface of each electrode. Two laminar flows were quickly established and could be separated prior to the outlets using the same spacer. 
The cell voltage and current were measured with a digital multimeter (Hewlett Packard, 34401A, USA). The solutions were introduced into the cell using a peristaltic pump (Ismatec, Switzerland, IPC 0-100 for Fig. 5, IPC-N-24 for the other figures) and tygon plastic tubes (internal diameter $=2.79 \mathrm{~mm}$ ). For the power experiments, the hot and cold solutions were both recirculated and thermostated in order to ensure the solution temperature stability over time. The hot reservoir was prevented from evaporation by using a glass coil. For all these experiments (except for Fig. 5 for which $T_{\text {cold }}$ corresponds to ambient temperature), the cold solution was refrigerated at a temperature fixed in the range of $10-12{ }^{\circ} \mathrm{C}$. The length between the thermostated reservoirs and the cell inlets was $40 \mathrm{~cm}$, the tubes of this part of the circuit being insulated by tissues and aluminium foils. The flow rate was obtained by collecting the solution at the outlet and measuring the mass over time. The concentrations of potassium ferricyanide and potassium ferrocyanide were equimolar and ranged from 1 to $200 \mathrm{mM}$. The concentration of sodium chloride was $100 \mathrm{mM}$. All chemicals were purchased from Fluka (Buchs, Switzerland) and used as received. The water was obtained from a Millipore (Milli-Q) purification system. The power measurements were performed after $10 \mathrm{~min}$ of open circuit conditions (resistance of the external electrical load, $R$, equivalent to infinite). Each point of the power curve was obtained by decreasing the resistance of the external load from $10 \mathrm{M} \Omega$ to $1 \Omega$, the measurement being performed $20 \mathrm{~s}$ after the corresponding resistance change. For the last experiment (long electrode with high concentration of the redox couple), this time was extended to $10 \mathrm{~min}$ in order to reach stability of the potential.

\section{Results and discussion}

\subsection{Numerical study (open circuit)}

In the experimental part, this general study of the thermal diffusion potential will be adapted to take into account a redox couple.

\subsubsection{Temperature distribution}

In Fig. 2 is shown the effect of the flow rate on the temperature difference between the electrodes on the channel walls. As the velocity is increased, the overall temperature difference increases, as the thermal diffusion does not extend to the channel walls. At the lowest velocity the system has time to equilibrate and consequently the temperature difference is zero. The case with the conductive spacer corresponds to the experimental configuration (the spacer is very thin $(60 \mu \mathrm{m})$ and its thermal diffusivity is of the same order of magnitude as that of water, i.e., $0.83 \times 10^{-7} \mathrm{~m}^{2} / \mathrm{s}$ compared with

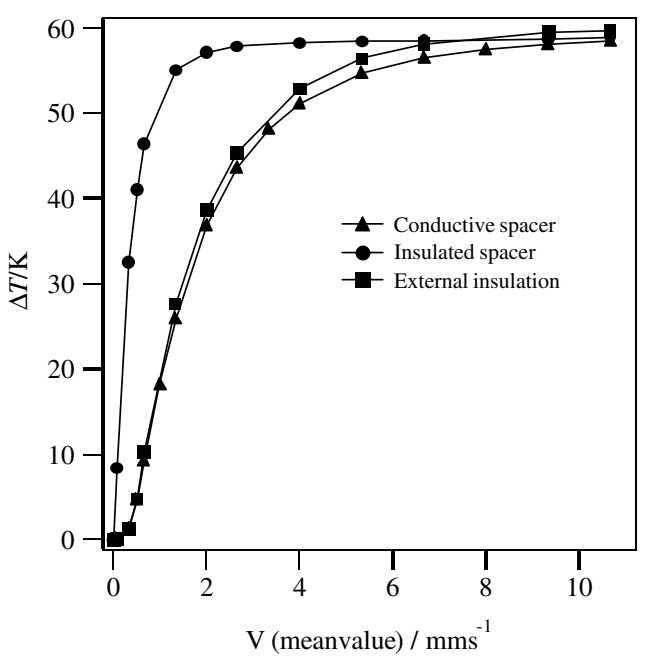

Fig. 2. Simulated temperature difference between the two electrodes versus the fluid average velocity, for conductive spacer, insulation spacer and external insulation, respectively.

$\left.1.43 \times 10^{-7} \mathrm{~m}^{2} / \mathrm{s}\right)$. The simulation incorporating a thermally insulating spacer shows that this configuration enables the maximum potential to be reached with a 3 times lower fluid velocity, which can be useful when the maximum flow rate given by the pump is limited as in the experiment described above. Fig. 2 also shows the effect of the external insulation of the cell (i.e., the case in which the external natural convection and radiation coefficients at the PMMA|air interface are reduced to $0.1 \%$ of their original value). In the present situation, the gain given by this insulation is low (from $2 \%$ to $6 \%$ depending on the flow rate) but this effect may be of importance in the case of cells having thin walls. This external heat exchange can be advantageous when the upper part of the cell is exposed to the sun.

The temperature distribution is shown in Fig. 3(a) for an intermediate flow rate value $\left(2 \mathrm{~mm} \mathrm{~s}^{-1}\right)$. It illustrates the temperature mixing due to the thermal conductivity of the spacer and the external cooling effect occurring at the upper part of the cell. The present hot temperature value $\left(80^{\circ} \mathrm{C}\right)$ corresponds to the limit of non-negligible thermal radiation (its flux value is here $2 / 3$ of the value of the external natural convection flux).

\subsubsection{Thermal diffusion potential}

Fig. 4 illustrates the transversal distribution of the thermal diffusion potential for different flow velocity mean values $(x=0.5 \mathrm{~cm})$. It confirms the increase in the potential difference when the thickness of the thermal boundary layer is decreased at high flow rates (note that this thermal boundary layer turns to a mixing layer after the fluid junction). It also appears that, due to the impact of the cold flux coming from the external exchange on the upper side of the cell (Fig. 3(a)), the potential decreases slightly at the surface of the upper electrode. This is also in accordance with the increase in the 
(a) Temperature $\left(\bar{V}=2 \mathrm{mms}^{-1}\right)$

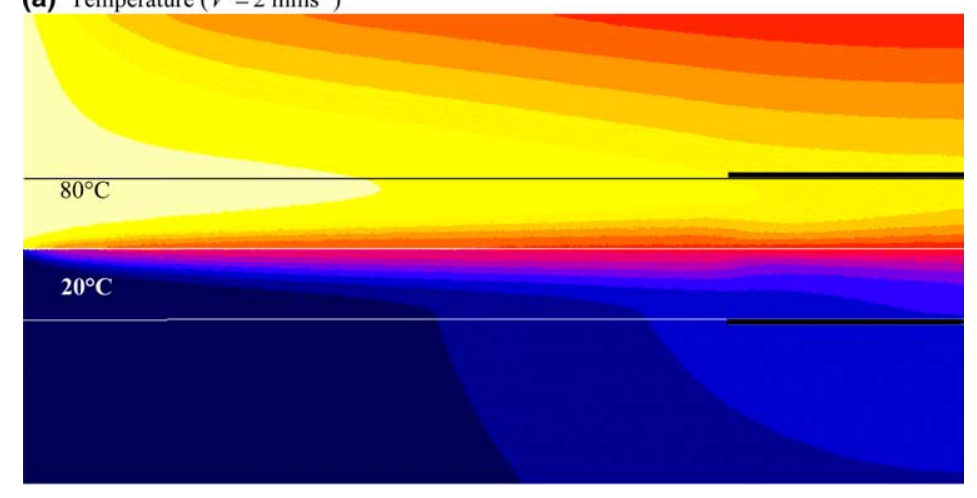

(b) Potential $\left(\bar{V}=2 \mathrm{mms}^{-1}\right)$

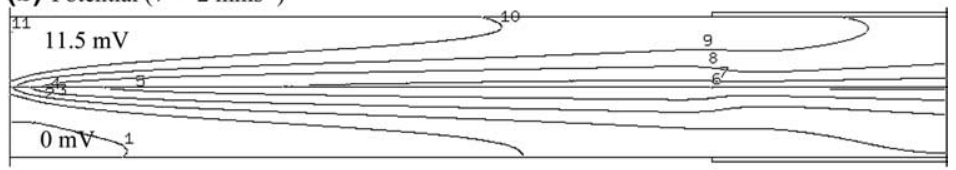

(c) Potential $\left(\bar{V}=10 \mathrm{mms}^{-1}\right)$

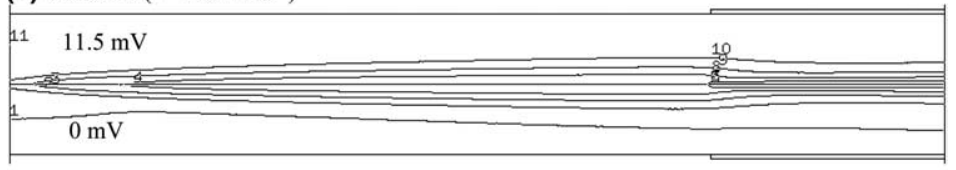

(d) Potential ( $\bar{V}=2 \mathrm{mms}^{-1} /$ insulating spacer $)$

$11.5 \mathrm{mV}$

Fig. 3. Isovalue representation for different flow rates and spacer type. (a) and (b) represent the respective temperature and electrical potential for an intermediate average velocity $\left(2 \mathrm{~mm} \mathrm{~s}^{-1}\right)$. The effect of a higher velocity $\left(10.7 \mathrm{~mm} \mathrm{~s}^{-1}\right)$ is illustrated in (c). For the intermediate velocity $\left(2 \mathrm{~mm} \mathrm{~s}{ }^{-1}\right)$, the effect of an insulating spacer to reduce the spreading of the potential gradient is shown in part (d). In total there are 14 colours between the indicated extremes on the figure (temperature) and 11 lines for the potential representations.

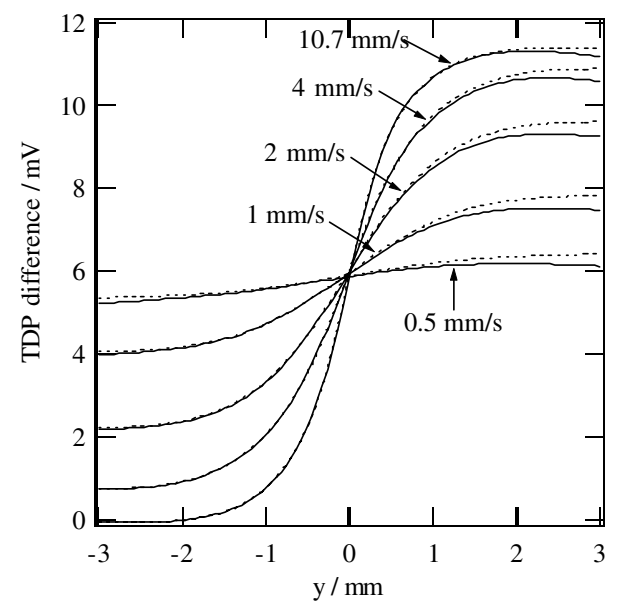

Fig. 4. Transversal distribution of the TDP for different flow velocity mean values (i.e., $0.5,1,2,4,10.7 \mathrm{~mm} \mathrm{~s}^{-1}$ ), at the middle of the electrode $(x=0.5 \mathrm{~cm}$ from its leading edge). Full line: including external heat exchange. Broken line: external insulation. temperature difference observed when the external insulation of the cell is taken into account (Fig. 2). Although this effect is small for the present system, it may be of importance for cells exposed to external forced convection.

Figs. 3(b)-(d) show the electrical potential isovalues in the simulated cross-section. As illustrated in Fig. 3(b), the simulated potential follows a similar pattern to the temperature on the conductive domain (fluid section). This finding is simply due to the direct relation between these parameters as described by Eqs. (2) and (11). It can be noted how the merging of two flows after the spacer narrows the temperature and potential isovalues between the electrodes. For the medium fluid velocity value chosen here $\left(\bar{V}=2 \mathrm{~mm} \mathrm{~s}^{-1}\right)$ the heat and potential "mixing layers" reach the electrodes, resulting in a potential difference lower than maximal as is also shown by the temperature difference in Fig. 2. Figs. 3(c) and (d) illustrate two ways of reaching the maximum potential for a given electrode position: (c) a higher velocity $\left(\bar{V}=10.7 \mathrm{~mm} \mathrm{~s}^{-1}\right)$ or (d) an insulating spacer. The sit- 
uation in Fig. 3(d) can also be obtained with a $\mathrm{Y}$ inlet junction of the fluids (instead of a spacer) or by placing the electrodes at the inlets of the channel. For this reason, the electrodes will be placed at the beginning of the channel for the second part of the experiments (power generation).

\subsection{Experimental study}

\subsubsection{Comparison with simulations (open circuit)}

In this part, a redox couple was added to in the solution (ferri/ferrocyanide redox couple) in order to extract a current, and thereby a power, from the temperature difference between the two flowing solutions. When the temperature of the liquids was varied, a linear dependence was found between the temperature difference and the open circuit potential, in agreement with Eq. (5). These findings confirm that the temperature difference can induce a power generating redox reaction at the electrodes. Recently, a similar study was carried out, taking advantage of a difference in ion concentration in the two flowing solutions [7]. The main driving force is here the variation of the standard potential of the redox couple with temperature [31-33]. Several reports exist on this effect; in one of them, the temperature effect on the redox potential of the ferri/ ferrocyanide redox couple was reported to be $-1.86 \mathrm{mV} /$ $\mathrm{K}$ [33]. For a temperature difference of $60 \mathrm{~K}$, this leads to a potential difference of $111 \mathrm{mV}$, which is much higher than the thermal diffusion potential (the thermal diffusion potential is on the order of $11 \mathrm{mV}$ as shown by Fig. 4 or calculated from the heats of transport if only $\mathrm{NaCl}$ is considered (Eq. (5)). According to these values, the resulting measured potential difference should have an absolute value of approximately $100 \mathrm{mV}$, which is higher than expected from the open circuit measurements shown in Fig. 5(a) (triangles). A precise comparison is difficult, as the maximum value of the measurable potential difference could not be attained for this electrode position (the required flow rate could not be reached with the peristaltic pump used, as shown in Fig. 4). This result is due to the fast thermal diffusion (which is about two orders of magnitude faster than typical molecular diffusion) and, as pointed out above, to the length and position of the electrodes (which are here placed $3 \mathrm{~mm}$ downstream of the fluid inlets). However, a direct comparison is hampered by the lack of information on heats of transport for the ferrocyanide and ferricyanide ions. Therefore, any comparison between simulations and experiments will have a purely qualitative nature.

In Fig. 5(a) is also shown the numerical TDP and the calculated potential difference based on Eq. (6) (i.e., including both the TDP and the effect on the redox properties deduced from the temperature profile in Fig. 2). It is apparent that the two curves corresponding
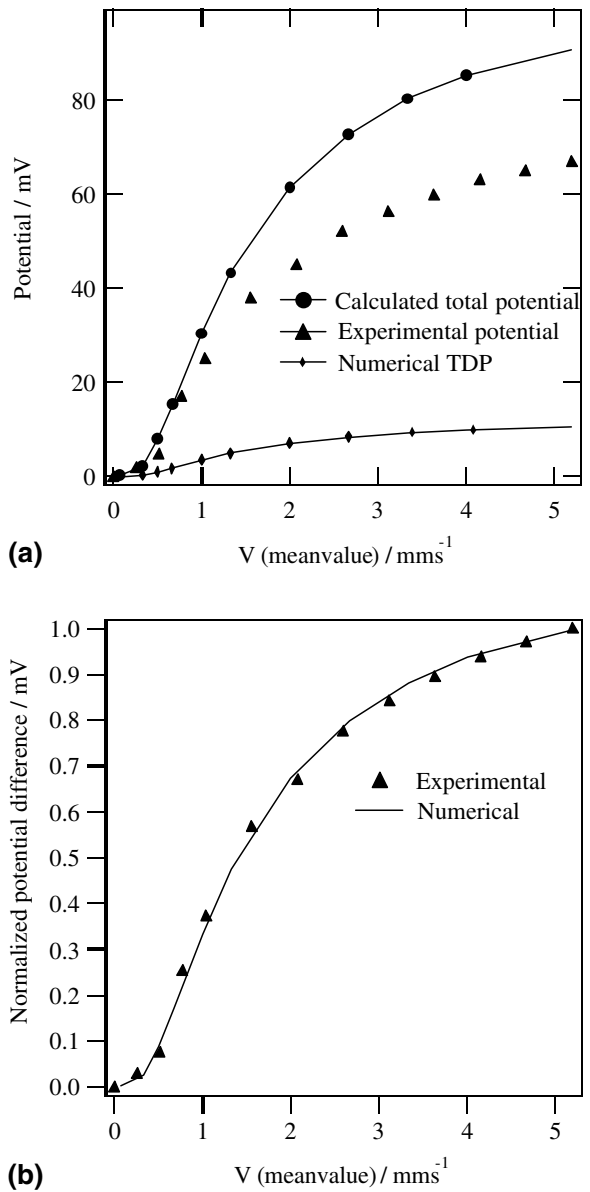

Fig. 5. All these curves correspond to $0.5 \mathrm{~cm}^{2}$ electrodes $(0.5 \times 1 \mathrm{~cm})$ placed at the middle of the channel under open circuit conditions (initial experimental configuration, also chosen for the simulation as described in Fig. 1). Temperatures are $20-80{ }^{\circ} \mathrm{C}$. (a) Triangles: overall absolute experimental value of the potential difference (i.e., contributions from the standard potential difference and TDP) measured between electrodes located at 1 and 4 in Fig. 1 at different flow rates, with a temperature difference of $60{ }^{\circ} \mathrm{C}$. Redox couple concentration is 50 mM. Diamonds: numerical TDP (based on simulated temperature fields and literature values of the heat of transport of $\mathrm{NaCl}$ ). Circles: calculated total numerical potential difference taking into account both the TDP and the temperature effect on the redox couple. All situations correspond to the case employing a conductive spacer. (b) Experimental and numerical total potential differences normalized by their values at $\bar{V}=5.2 \mathrm{~mm} \mathrm{~s}^{-1}$ (maximum velocity of the experimental range).

to the numerical and experimental overall potential difference do not coincide. This finding is not surprising since the TDP contributions from ferri-, ferrocyanide and potassium have not been considered. Another point concerns the temperature dependence on the standard redox potential; in fact this coefficient is likely always to be hampered to some extend by a TDP. However, the relative values of the potential may provide a good measure of the quality of the thermal simulations and allow a comparison of the flow rate effect on the thermal transfer. This is the idea behind Fig. 5(b) in which the potential differences have been normalized by their value 
at a flow rate of $5.2 \mathrm{~mm} \mathrm{~s}^{-1}$. The good agreement between the normalized numerical and experimental values emphasizes that the numerical model can successfully model the temperature distribution in the system.

The curvature of the potential evolution for low flow rates can be explained by the gap between the electrodes and the channel inlet. The greater this gap, the flatter is the potential increase at low flow rates. Indeed, due to this gap and the diffusive mixing, a minimum fluid velocity is needed to maintain the inlet temperature difference until the electrodes are reached. For greater velocities, the potential increase is much faster, due to the short electrode length compared to the gap. After the temperature gradient has reached the end of the electrodes, the maximum potential difference is reached, leading to the observed asymptote and inflexion point.

\subsubsection{Power generation}

When electrodes placed on the walls of the flow cell were connected through an external load, a current could be measured in the external circuit. In Fig. 6(a) are shown the potential differences and the deduced power values versus the measured current, for different concentrations of the ferri/ferrocyanide redox couple (equimolar values ranging from 1 to $200 \mathrm{mM}$ ). Following the simulation results, the $0.5 \mathrm{~cm}^{2}$ electrodes are here placed at the entry of the channel to increase the performance of the system for a given flow rate value (here $\bar{V}=2.3 \mathrm{~mm} \mathrm{~s}^{-1}$ ). As illustrated in Fig. 6(b), the maximum value of the generated power follows the expected linear dependence versus the redox couple concentration, in the present concentration range (the maximum concentration value being limited by the precipitation of the redox species in the refrigerated reservoir). The observed shift to higher currents is explained by the increase of the diffusion limiting current with the redox couple concentration. As expected, the same phenomenon is observed when the flow rate is increased (Fig. 7). These experiments show that the optimum velocity is lower than for previous experiments (Fig. 5), due to the present position of the electrodes at the channel inlet (no gap).

The effect of a larger electrode surface is presented in Fig. $8\left(2.5 \mathrm{~cm}^{2}\right.$ surface corresponding to a 5 times longer electrode, i.e., $5 \times 50 \mathrm{~mm}$ ), for $\Delta T=55^{\circ} \mathrm{C}, 0.2 \mathrm{M}$ of redox couple concentration and $\bar{V}=4.6 \mathrm{~mm} \mathrm{~s}^{-1}$. One may note that the difference with the previous maximum pump value of $5.2 \mathrm{~mm} \mathrm{~s}^{-1}$ is due to the thicker spacer. The maximum power value reaches $200 \mu \mathrm{W}$, leading to the expected gain of 5 (compared to the $0.5 \mathrm{~cm}^{2}$ electrodes placed at the entry of the channel giving near to $40 \mu \mathrm{W}$ for the same velocity, as shown in Fig. 7). The resulting order of magnitude of reachable power density is $0.1 \mathrm{~mW} / \mathrm{cm}^{2}$ for a temperature difference of $55{ }^{\circ} \mathrm{C}$ between the solutions in the cell. One of the conditions
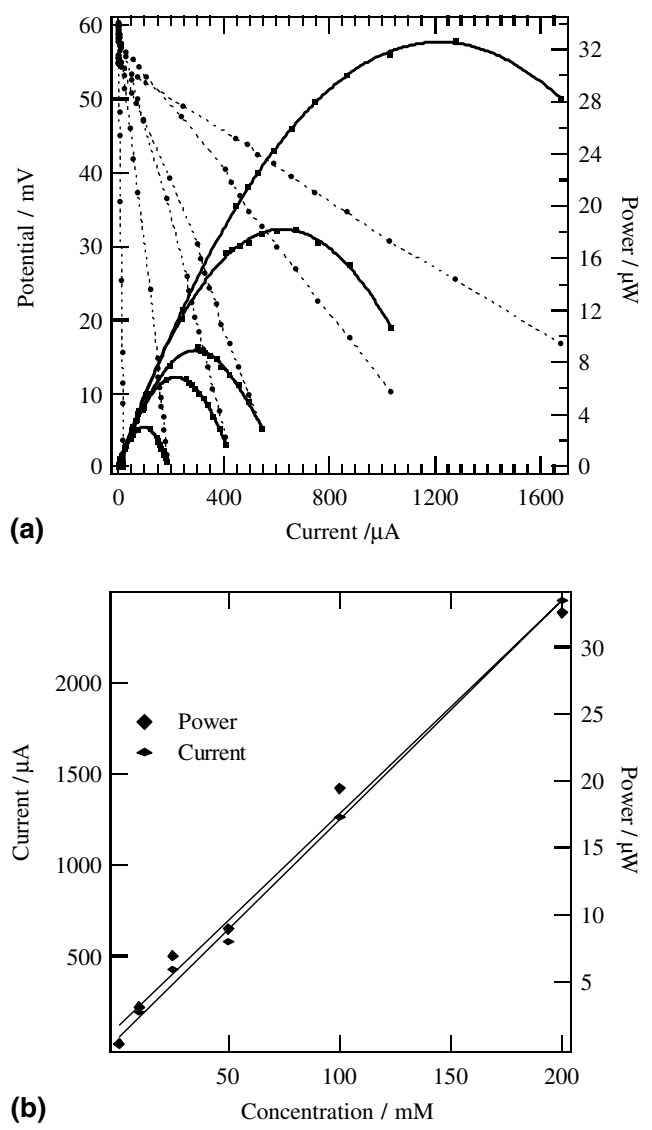

Fig. 6 . The $0.5 \mathrm{~cm}^{2}$ electrodes were placed at the entry of the channel to increase the performance of the system for a given flow rate value $\left(\bar{V}=2.3 \mathrm{~mm} \mathrm{~s}^{-1}\right)$. (a) Experimentally measured potential differences and power values versus current values for different ferricyanide and ferrocyanide concentrations (equimolar values of 1, 10, 25, 50, 100 and $200 \mathrm{mM}$ ). Sodium chloride concentrations were $100 \mathrm{mM}$. Each curve was obtained by decreasing the external load value from $10 \mathrm{M} \Omega$ to $1 \Omega$. Temperature difference is $55^{\circ} \mathrm{C}$. (b) Evolution of the maximum power value versus the redox couple concentration.

for a scaling to meter square surfaces (i.e., $1 \mathrm{~W} / \mathrm{cm}^{2}$ ) is to work with a centimetre scale channel width in order to ensure the non-mixing condition along all the electrode length with a reasonable pressure drop of the flow. The present value of power density is the same as that obtained in [8] in a rotating cell with $\Delta T=50{ }^{\circ} \mathrm{C}$ and the same same redox couple concentration $(0.2 \mathrm{M})$. These results are also in the range given in [6], i.e., $0.3-2 \mathrm{~W} / \mathrm{m}^{2}$, for the same kind of flow cell with the same redox couple concentration and a temperature difference of $40^{\circ} \mathrm{C}$ (the first value being experimental and the second being the prediction without mass transfer limitations). Higher values of power density could be reached, for a given "floor space" of the electrodes, by using a multilayer channel and electrode assembly.

In order to estimate the overall yield, the energy required to pump the solutions through the cell and the energy required to heat one solution should be considered. The kinetic energy is $0.5 m_{\mathrm{w}} \bar{V}^{2}$, where $m_{\mathrm{w}}$ is the 


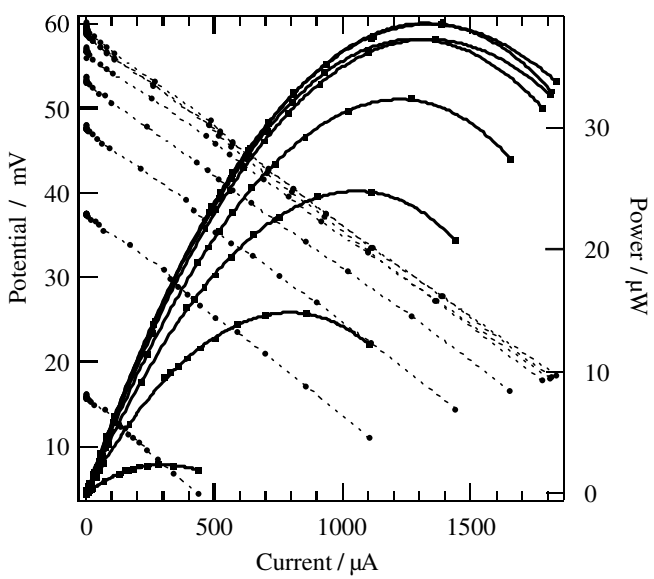

Fig. 7. Effect of the fluid flow rate on the potential difference and power measurements. Flow rates range from 2 to $16 \mathrm{ml} / \mathrm{min}$ with $2 \mathrm{ml} /$ min increments, corresponding to $\bar{V}$ values comprised between and 0.575 and $4.6 \mathrm{~mm} \mathrm{~s}^{-1}$. The 3 first intermediate values before reaching the plateau of maximum power are $1.15,1.73$ and $2.3 \mathrm{~mm} \mathrm{~s}^{-1}$. Redox couple concentration is $200 \mathrm{mM}$ and temperature difference $55^{\circ} \mathrm{C}$.

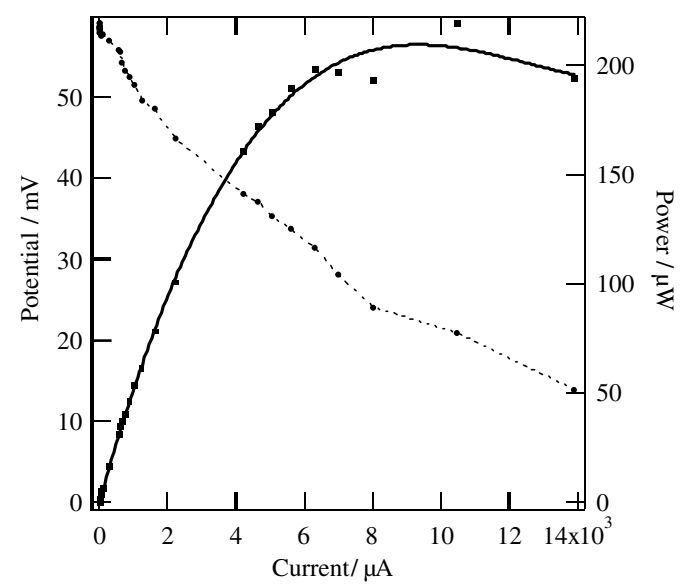

Fig. 8. Experimental potential differences and power values versus current values for $2.5 \mathrm{~cm}^{2}$ electrodes $(5 \times 50 \mathrm{~mm}$ dimensions $)$ and 200 $\mathrm{mM}$ ferricyanide and ferrocyanide concentrations. Temperature difference is $55^{\circ} \mathrm{C} . \bar{V}=4.6 \mathrm{~mm} \mathrm{~s}^{-1}$.

mass of water. The power (energy per unit time) can be expressed in terms of the mass flow rate, $V_{\mathrm{m}}$ as: $0.5 V_{\mathrm{m}}$ $\bar{V}^{2}$. Using the experimental mass flow rate of $0.3 \mathrm{~g} / \mathrm{s}$ $\left(\bar{V}=5.2 \mathrm{~mm} \mathrm{~s}^{-1}\right)$, an estimated pump power of $1.5 \mathrm{nW}$ can be estimated.

The power required to heat the solution, $Q_{\text {heat }}$, can be estimated as: $Q_{\text {heat }}=m_{\mathrm{w}} C_{\mathrm{p}} \Delta T$, where $C_{\mathrm{p}}$ is the specific heat capacity of water. The power required to heat the solution, $P_{\text {heat }}$, is given by: $P_{\text {heat }}=1 / 2 V_{\mathrm{m}} C_{\mathrm{p}} \Delta T$ (only half of the solution needs to be heated). Using $V_{\mathrm{m}}=0.15 \mathrm{~g} / \mathrm{s}$ (using a channel depth identical to the spacer and electrodes depth, i.e., $5 \mathrm{~mm}$, corresponding to half the present experimental channel depth) and using $C_{\mathrm{p}}=4.18 \mathrm{~J} \mathrm{~g}^{-1} \mathrm{~K}^{-1}$ with $\Delta T=55 \mathrm{~K}$, the result is 17.3 $\mathrm{W}$. The power required to pump the solution can thus be neglected and we arrive at an approximate efficiency (see
Fig. 6(a)) of: $200 \mu \mathrm{W} / 17 \mathrm{~W} \approx 10^{-30} \%$. Obviously this efficiency yield is extremely low, but it should be stressed that an efficient conversion yield was not the aim of this system. It is simply an illustration of the ability to sample energy in places where solutions of elevated temperature are flowing in parallel under laminar conditions (i.e., extraction of a small fraction of the energy instead of a conversion, as the main part of the heat content flows away). When the principle of electrochemical thermocouples is used in stationary systems (employing a cation exchange membrane) higher conversion efficiencies are obtained (i.e., in the order of $0.1 \%$ ) [11]. The advantages of the present system are that no membrane is required, as in $[4-6,9,34]$ and that the system can be installed in places where flowing solutions of different temperatures are already present.

\section{Conclusions}

The hydrovoltaic cell principle, based on concentration diffusion potentials, has been extended to consider thermal effects. The numerical simulations (heat transfer and open circuit thermal diffusion potential) and the experimental results using a redox couple confirm that hydrovoltaic cells in principle can be used to generate electricity from low-grade heat sources. The experimental and numerical studies are shown to be in good agreement concerning the influence of the flow rate on the thermal transfer. For the resulting measured potential difference (mixed contribution of TDP and redox couple effect), the agreement is qualitatively good.

Due to its very low energetic efficiency, this system has to be seen as a thermal energy sampler (rather than a converter). The generated power per electrode surface is low $\left(1 \mathrm{~W} / \mathrm{m}^{2}\right)$ but, from further additional developments, one can envision the use of this concept in more practical setups, such as multi-layer systems. Finally, the temperature modelling outlined in this paper can also be used to quantify unwanted temperature induced artefacts in (micro) analytical systems.

\section{Acknowledgements}

The authors thank the Ecole Polytechnique Fédérale de Lausanne for continuous support of the Laboratoire d'Electrochimie Physique et Analytique.

\section{Appendix A}

Eqs. (11) and (12) are derived in the global general form (A.1) and (A.2), using the Galerkin formulation (multiplication by a projective function $\alpha$ and 
integration on the domain of study, $\Omega$ ), where $i$ design are the 1,2 species:

$$
\begin{aligned}
& \int_{\Omega} \int \alpha\left[\frac{\partial c_{i}}{\partial t}+\nabla \cdot\left(-D_{i} \nabla c_{i}+V c_{i}-\frac{z_{i} F}{R T} D_{i} c_{i} \nabla \phi\right.\right. \\
& \left.\left.-\frac{{ }^{*} Q_{i}}{R T^{2}} D_{i} c_{i} \nabla T\right)\right] \mathrm{d} \Omega=0, \quad i=1,2, \\
& \int_{\Omega} \int \alpha\left[z_{1} c_{1}-z_{2} c_{2}\right] \mathrm{d} \Omega=0 .
\end{aligned}
$$

By decomposing the product between $\alpha$ and the divergence, the second-order derivative of (A.1) and (A.2) is

$\alpha \nabla \cdot\left(-D_{i} \nabla c_{i}\right)=\nabla \cdot\left(-\alpha D_{i} \nabla c_{i}\right)+D_{i} \nabla \alpha \cdot \nabla c_{i}$.

Injecting (A.3) in (A.1) and (A.2) and using the Ostrogradsky theorem, the divergence term is rejected at the boundary (A.4), where it expresses the flux conditions of the species which here equals zero (no flux at the boundaries of the domain)

$$
\begin{aligned}
& \iint_{\Omega}\left[\alpha \frac{\partial c_{i}}{\partial t}+D_{i} \nabla \alpha \cdot \nabla c_{i}+\alpha \boldsymbol{V} \nabla c_{i}+\frac{z_{i} F}{R T} D_{i} c_{i} \nabla \alpha \cdot \nabla \phi\right. \\
& \left.\quad+\frac{{ }^{*} Q_{i}}{R T^{2}} D_{i} c_{i} \nabla \alpha \cdot \nabla T\right] \mathrm{d} \Omega=0
\end{aligned}
$$

Applying (A.4) to (A.1) and (A.2), and writing it in a matrix form, we obtain (A.5), where $\beta$ is the interpolation function of the unknown vector $\left[c_{1}, c_{2}, \phi, T\right]$

$$
\begin{aligned}
& {\left[\begin{array}{ccc}
\alpha & 0 & 0 \\
0 & \alpha & 0 \\
0 & 0 & 0
\end{array}\right] \cdot\left[\begin{array}{l}
\frac{\partial c_{1}}{\partial t} \\
\frac{\partial c_{2}}{\partial t} \\
\frac{\partial \phi}{\partial t}
\end{array}\right]} \\
& +\left[\begin{array}{cc}
D_{1} \nabla \alpha \nabla \beta+\boldsymbol{V} \alpha \beta & 0 \\
0 & D_{2} \nabla \alpha \nabla \beta+\boldsymbol{V} \alpha \beta \\
\alpha z_{1} \beta & -\alpha z_{2} \beta \\
0 & 0
\end{array}\right. \\
& \left.\begin{array}{cc}
\frac{z_{1} F}{R T} D_{1} c_{1} \nabla \alpha \nabla \beta & \frac{{ }^{*} Q_{1}}{R T^{2}} D_{1} c_{1} \nabla \alpha \nabla \beta \\
\frac{z_{2} F}{R T} D_{2} c_{2} \nabla \alpha \nabla \beta & \frac{{ }^{2} Q_{2}}{R T^{2}} D_{2} c_{2} \nabla \alpha \nabla \beta \\
0 & 0 \\
0 & D \nabla \alpha \nabla \beta+\boldsymbol{V} \alpha \beta
\end{array}\right] \\
& {\left[\begin{array}{l}
c_{1} \\
c_{2} \\
\phi \\
T
\end{array}\right]=0}
\end{aligned}
$$

\section{Appendix B}

Due to the high value of the thermal diffusivity (compared to the diffusion coefficients of the species in solution) and in order to maintain a temperature gradient, the flow velocity value and the channel thickness are chosen larger than in a previous study [25]. As a consequence, the diffusion coefficients of the species $D_{1}$ and $D_{2}\left(1.33 \times 10^{-9} \mathrm{~m}^{2} / \mathrm{s}\right.$ for $\mathrm{Na}^{+}$and $2.03 \times 10^{-9} \mathrm{~m}^{2} / \mathrm{s}$ for $\mathrm{Cl}^{-}$) are artificially increased by 2 orders of magnitude in order to keep the mesh Peclet number at a reasonable value. Indeed, real $D$ values would imply a mesh size that cannot be solved with our computer resources. It has been verified that this scaling has no effect on the electrical potential results. It can be easily explained by the fact that the diffusion coefficient is present in both the thermal transport and migration terms of Eq. (11), the latter balancing the thermal one at each time. Consequently, for a given geometry and species, the main parameters governing the temperature and potential distributions are the thermal diffusivity $\lambda / \rho C_{\mathrm{p}}$ and the flow rate. In order to verify the model and the approximations in Eq. (11) it was confirmed that simulation of the thermal diffusion potential results in a similar value as obtained from Eq. (5).

\section{References}

[1] R. Haase, Thermodynamics of Irreversible Processes, AddisonWesley, New York, 1969.

[2] H.H. Girault, J. Chem. Soc. Faraday Trans. 84 (1988) 2147.

[3] T. Osakai, H. Ogawa, T. Ozeki, H.H. Girault, J. Phys. Chem. B 107 (2003) 9829.

[4] R. Ferrigno, A.D. Strook, T.D. Clark, M. Mayer, G.M. Whitesides, J. Am. Chem. Soc. 124 (2002) 12930.

[5] R. Ferrigno, A.D. Strook, T.D. Clark, M. Mayer, G.M. Whitesides, in: 53rd Annual Meeting of the International Society of Electrochemistry, Düsseldorf, Germany, 2002.

[6] J.M. Hornut, A. Storck, J. Appl. Electrochem. 21 (1991) 1103.

[7] G. Lagger, H. Jensen, J. Josserand, H.H. Girault, J. Electroanal. Chem. 545 (2003) 1.

[8] B. Burrows, J. Electrochem. Soc. 123 (1976) 154.

[9] T.I. Quickenden, Y. Mua, J. Electrochem. Soc. 142 (1995) 3985.

[10] A.V. Sokirko, Electrochim. Acta 39 (1994) 597.

[11] T. Hirai, K. Shindo, T. Ogata, J. Electrochem. Soc. 143 (1996) 1305.

[12] J.N. Agar, in: P. Delahay (Ed.), Advances in Electrochemistry and Electrochemical Engineering, vol. 3, 1963, p. 31.

[13] S. Hardt, W. Ehrfeld, V. Hessel, K.M. Vanden Bussche, Chem. Eng. Commun. 190 (2003) 540.

[14] A.A. Darhuber, J.M. Davis, S.M. Troian, W.W. Reisner, Phys. Fluids 15 (2003) 1295.

[15] N. Damean, P.P.L. Regtien, M. Elwenspoek, Sensors Actuators A 105 (2003) 137.

[16] Y. Fang, W.W. Liou, J. Heat Transfer 124 (2002) 338.

[17] M.N. Slyadnev, Y. Tanaka, M. Tokeshi, T. Kitamori, Anal. Chem. 73 (2001) 4037.

[18] A.I.K. Lao, T.M.H. Lee, I.M. Hsing, N.Y. Ip, Sensors Actuators A 84 (2000) 11.

[19] E.W. Kreutz, N. Pirch, T. Ebert, R. Wester, B. Ollier, P. Loosen, R. Poprawe, Microelectron. J. 31 (2000) 787.

[20] L.N. Jiang, Y.L. Wang, M. Wong, Y. Zohar, J. Micromech. Microeng. 9 (1999) 422.

[21] C. Alepee, R. Maurer, L. Paratte, L. Vulpescu, P. Renaud, A. Renken, Microreaction technology: industrial prospects, in: Proceedings of the International Conference on Microreaction Technology, third ed., Frankfurt, April 18-21, 1999, p. 514. 
[22] K.F. Jensen, I.M. Hsing, R. Srinivasan, M.A. Schmidt, Microreaction technology, in: Proceedings of the International Conference on Microreaction Technology, first ed., Mainz, February 23-25, 1997, 1998, p. 2.

[23] E. Burian, D. Pogany, T. Lalinsky, N. Seliger, E. Gornik, Sensors Actuators A 68 (1998) 372.

[24] N.T. Nguyen, D. Bochnia, R. Kiehnscherf, W. Doetzel, Sensors Actuators A 55 (1996) 49.

[25] J. Josserand, G. Lagger, H. Jensen, R. Ferrigno, H.H. Girault, J. Electroanal. Chem. 546 (2003) 1.

[26] J.S. Newman, Electrochemical Systems, Prentice-Hall, Englewood Cliffs, NJ, 1991.

[27] F.K. Incropera, D.P. Dewitt, Fundamentals of Heat and Mass Transfer, Wiley, New York, 1990.
[28] H. Jensen, V. Devaud, J. Josserand, H.H. Girault, J. Electroanal. Chem. 537 (2002) 77.

[29] R.A. Robinson, R.H. Stokes, Electrolyte Solutions, Butterworths, London, 1959.

[30] H.F. Mark, N. Bikales, C.G. Overberger, G. Menges, J.I. Kroschwitz, Encyclopedia of Polymer Science and Engineering, Wiley, New York, 1987.

[31] W.A. Eaton, P. George, G.I.H. Hanania, J. Phys. Chem. 71 (1967) 2016.

[32] G.I.H. Hanania, D.H. Irvine, W.A. Eaton, P. George, J. Phys. Chem. 71 (1967) 2022.

[33] Y. Liu, L.C. Seefeldt, V.D. Parker, Anal. Biochem. 250 (1997) 196.

[34] T.I. Quickenden, Y. Mua, J. Electrochem. Soc. 142 (1995) 3652. 